\title{
Mitochondrial Dysfunction Induced by Fatty Acid Ethyl Esters, Myocardial Metabolites of Ethanol
}

\author{
Louis G. LANGe and BURTON E. Sobel, Cardiovascular Division, Washington \\ University School of Medicine, St. Louis, Missouri 63110
}

A B S T R A C T Mechanisms responsible for alcohol-induced heart muscle disease have been difficult to elucidate partly because of previously obscure, demonstrable cardiac metabolism of ethanol. Recently, fatty acid ethyl esters were identified in our laboratory and found to be myocardial metabolites of ethanol. In the present study, they have been shown to induce mitochondrial dysfunction. Incubation of isolated myocardial mitochondria with fatty acid ethyl esters led to a concentration-dependent reduction of the respiratory control ratio index of coupling of oxidative phosphorylation and decrement of maximal rate of oxygen consumption. Furthermore, fatty acid ethyl esters were demonstrated to bind to mitochondria in vitro, and, importantly, $72 \%$ of intracellularly synthesized ethyl esters were found to bind to mitochondria isolated from intact tissue incubated with ethanol. Protein binding of fatty acid ethyl esters was markedly less than that of fatty acids. Because uncoupling of mitochondrial oxidative phosphorylation correlated with the cleavage of fatty acid ethyl ester shown to be initially bound to mitochondria, with resultant generation of fatty acid, a potent uncoupler, in a locus in or near the mitochondrial membrane, fatty acid ethyl esters may contribute to a potentially toxic shuttle for fatty acid with transport from physiological intracellular binding sites to the mitochondrial membrane; direct effects of fatty acid ethyl esters may also be deleterious. Operation of this shuttle as a result of ethanol ingestion and subsequent accumulation of fatty acid ethyl esters may account for the impaired mitochondrial function and inefficient energy production associated with toxic effects of ethanol on the heart.

Address all correspondence to Dr. L. G. Lange.

Received for publication 13 December 1982 and in revised form 2 March 1983.

\section{INTRODUCTION}

Alcohol-induced heart disease is a common disorder afflicting more than 200,000 Americans (1). Despite its prevalence, its well-characterized ethanol-induced depression of myocardial contractility $(2-4)$ and arrhythmias (5), and changes that it produces in myocardial lipid composition (6-8), the pathophysiology of this disorder has remained obscure. The apparent lack of demonstrable cardiac metabolism of ethanol $(6,9)$ has made it difficult to elucidate a direct link between exposure to alcohol and the metabolic changes noted.

It has been known since 1917 that even moderate consumption of alcohol increases the basal metabolic rate of some subjects, which is reflected by a 5-7\% increase in total body oxygen consumption (10). Such changes could be due to alterations in mitochondrial function. Consumption of alcohol over several weeks leads to gross morphological changes in heart mitochondria in laboratory animals $(11,12)$ and human subjects $(13,14)$. Impairment of mitochondrial function has been noted as well and it includes diminished phosphate/oxygen ratios $(\mathrm{P} / \mathrm{O})^{1}$ and respiratory control ratios (15-18); these are changes that do not occur after exposure of mitochondria to ethanol in vitro.

Recent work from our laboratory has identified a class of metabolites of ethanol metabolism that are formed in isolated, perfused rabbit hearts (19). The products, fatty acid ethyl esters, arise principally through a soluble esterase-catalyzed esterification of ethanol with free fatty acid (20). The reaction is prominent at physiological concentrations of each substrate. Subsequently, fatty acid ethyl esters in concentrations as high as $115 \mu \mathrm{M}$ were identified in hearts obtained

\footnotetext{
${ }^{1}$ Abbreviations used in this paper: $\mathrm{HM}$, homogenizing medium; $\mathrm{P} / \mathrm{O}$, phosphate/oxygen ratio.
} 
at autopsy from patients either acutely intoxicated at the time of death or chronically exposed to alcohol (21).

The present study was undertaken to determine whether fatty acid ethyl esters could be the metabolic link between ethanol consumption and alcohol-induced mitochondrial dysfunction. Results with isolated heart muscle mitochondria from rabbits indicate that fatty acid ethyl esters may initiate a toxic fatty acid shuttle which leads to impairment and uncoupling of oxidative phosphorylation.

\section{METHODS}

All reagents were of the highest grade commercially available. Lipid solvents were distilled before use. Lipids were purified by standard thin-layer chromatographic techniques $(19,20)$. Radiolabeled compounds were purchased from Sigma Chemical Co., St. Louis, MO or Amersham Corp., Arlington Heights, IL. Ethyl $\left[{ }^{3} \mathrm{H}\right]$ oleate was a gift of Dr. S. Mogelson.

Preparation of mitochondria. Fresh mitochondria were prepared daily from rabbit hearts by standard methods (2224). Oxidative function was assessed polarographically with a polarograph (Yellow Springs Instrument Co., Yellow Springs, $\mathrm{OH}$; reference 24, 25). Rabbits were sacrificed after cervical dislocation and their hearts were removed immediately and placed in homogenizing medium (HM): $0.25 \mathrm{M}$ sucrose, $10 \mathrm{mM}$ EDTA, $0.1 \%$ bovine serum albumin (BSA) at $0-4^{\circ} \mathrm{C}$. The preparation was completed as rapidly as possible. After the trimmed ventricles had been washed with HM, the tissue was minced with a scissors and passed through a precooled, hand-operated muscle press to give a $15 \%$ (wt/ vol) suspension which was homogenized in a Potter-Elvehjen apparatus (Fisher Scientific Co., Pittsburgh, PA) (six strokes). After filtration through cheesecloth, the suspension was centrifuged at $2,500 \mathrm{~g}$ for $10 \mathrm{~min}$. The supernatant fat on the surface was removed with a cotton swab, the pellet discarded, and the supernatant fraction centrifuged at 9,000 $g$ for $10 \mathrm{~min}$. The resulting mitochondrial pellet was washed three times with $30 \mathrm{ml}$ each of $\mathrm{HM}$ and repelleted at 9,000 $g$ for $10 \mathrm{~min}$. The final pellet was gently resuspended in 1 $\mathrm{ml} \mathrm{HM} / \mathrm{g}$ original wet weight of ventricle.

The mitochondrial preparation was characterized polarographically, microscopically, and enzymatically. Electron microscopy of the final mitochondrial pellet after fixation, staining, and processing as previously described (26) demonstrated that in low-power views $(\times 3,000)$, essentially all formed elements were mitochondria; only a small amount of disrupted mitochondrial components was evident. Assay of an aliquot of mitochondria equivalent to that used for polarographic assays detected essentially no enzymatic activity for cleavage of cholesterol $\left[{ }^{14} \mathrm{C}\right]$ oleate (lysosomal marker) or synthesis of triglyceride from $\left[{ }^{14} \mathrm{C}\right]$ oleoyl Coenzyme A (microsomal marker).

Polarographic assay with a recording oxygen electrode was performed in incubation medium consisting of $250 \mu \mathrm{lmi}$ tochondrial suspension, $250 \mu \mathrm{l} \mathrm{HM}$, and $2.0 \mathrm{ml}$ medium containing $225 \mu \mathrm{mol}$ potassium chloride, $150 \mu \mathrm{mol}$ Tris, 37.5 $\mu \mathrm{mol}$ potassium monohydrogen phosphate, $15 \mu \mathrm{mol}$ magnesium chloride, and $20 \mu \mathrm{mol}$ EDTA, at $\mathrm{pH} 7.4$, at a final mitochondrial protein concentration of $2 \mathrm{mg} / \mathrm{ml}(27)$. State III respiration was initiated by addition of $700 \mu \mathrm{l}$ of a solution of sodium malate-sodium pyruvate, $\mathrm{pH} 7$, to give a final concentration of $21 \mathrm{mM}$; this was followed after $2 \mathrm{~min}$ by addition of ADP, $1.25 \mu \mathrm{mol}$ in a volume of $50 \mu \mathrm{l}$. Recordings were performed at least in duplicate with calculations of the rates of oxygen consumption obtained from the second ADP addition. Quality of the preparation was assured by determination of respiratory control indexes and the rate of state III oxygen consumption as previously described. Preparations exhibiting coupling ratios of $<4.0$ or rates of maximum oxygen consumption less than 180 natoms/min per $\mathrm{mg}$ were not used. Over many months, mitochondrial preparations had coupling ratios of 7 to 9 , maximal oxygen consumption rates of 220 natoms/min per $\mathrm{mg}$, and $\mathrm{P} / \mathrm{O}$ ratios of 2.7 to 3.0. The percentage of uncoupling was calculated as: $[100 \%$ - $($ CRC - 1)]/[(CRC control - 1) X 100], where CRC - 1 is the observed coupling ratio minus 1.0 , and CRC control -1 is the control coupling ratio minus 1 .

Incubation of mitochondria with fatty acid ethyl esters. Most experiments examining the effect of fatty acid ethyl esters on isolated mitochondria were performed with ethyl oleate incorporated into unilamellar vesicles of egg phosphatidylcholine to avoid phase separations which were noted with fatty acid ethyl esters at higher concentrations ( $>50$ $\mu \mathrm{M}$ ). Vesicles were prepared by co-evaporation of a mixture of ethyl oleate at selected concentrations up to $0.2 \mu \mathrm{mol}$ and egg phosphatidylcholine, $10 \mu \mathrm{mol}$, in chloroform/methanol (2:1). After adding $2 \mathrm{ml}$ of incubation medium and vortexing, the lipid preparation was sonified at $70 \mathrm{~W}$ with a sonifyer (Branson Sonic Power Co., Danbury, CT) for $15 \mathrm{~min}$ at $4^{\circ} \mathrm{C}(28)$, and then centrifuged at $50,000 \mathrm{~g}$ for $60 \mathrm{~min}$ to yield a very faintly opalescent supernatant of ethyl oleate vesicles. Concentrations were estimated by recovery of added ethyl $\left[{ }^{3} \mathrm{H}\right]$ oleate. Analysis of the final preparation indicated that free fatty acid comprised $<0.25 \%$ of the ethyl ester concentration.

In experiments examining the effect of ethyl oleate on respiratory function of isolated mitochondria, incubations were carried out at $25^{\circ} \mathrm{C}$, unless otherwise stated. Medium contained pyruvate, $21 \mathrm{mM}$; malate, $21 \mathrm{mM}$; and ADP, 390 $\mu \mathrm{M}$, to ensure stability of the mitochondria. Control incubations were performed with phosphatidylcholine vesicles that did not include ethyl esters. After preincubation for 15$120 \mathrm{~min}$, suspensions containing isolated mitochondria and vesicles with or without ethyl ester were centrifuged at 9,000 $g$ for $10 \mathrm{~min}$ at $4^{\circ} \mathrm{C}$. The resultant mitochondrial pellet was resuspended in fresh incubation medium. Respiratory function was assessed polarographically. In other experiments, resuspended mitochondria were extracted into lipid solvents, and the amount of bound ethyl $\left[{ }^{3} \mathrm{H}\right]$ oleate and $\left[{ }^{3} \mathrm{H}\right]$ oleate per milligram mitochondrial protein was determined after thinlayer chromatography on silica gel developed with petroleum ether/diethyl ether/acetic acid (75:5:1) (19).

In other experiments, suspensions of isolated mitochondria incubated with ethyl $\left[{ }^{3} \mathrm{H}\right]$ oleate vesicles were extracted before centrifugation. The amount of $\left[{ }^{3} \mathrm{H}\right]$ oleate released was quantified and expressed as nanomoles of $\left[{ }^{3} \mathrm{H}\right]$ oleate released per minute per milligram mitochondrial protein. Recoveries $(70-80 \%)$ were corrected for extraction, and chromatographic losses were assessed in parallel procedures with labeled oleate which ranged from 20 to $30 \%$.

Protein binding of ethyl oleate. To assess the relative binding affinities of ethyl $\left[{ }^{3} \mathrm{H}\right]$ oleate and $\left[{ }^{14} \mathrm{C}\right]$ oleate to BSA, equimolar mixtures of the two labeled species, $10 \mu \mathrm{M}$, were incubated with defatted albumin, $29 \mu \mathrm{M}$, at $25^{\circ} \mathrm{C}$ for $10 \mathrm{~min}$ in a total volume of $1 \mathrm{ml}$. Then this mixture was gel filtered through Sephadex G-50, $1.6 \times 30 \mathrm{~cm}$, equilibrated at $25^{\circ} \mathrm{C}$ with mitochondrial incubation medium. Fractions of $1 \mathrm{ml}$ each were collected at a flow rate of $25 \mathrm{ml} / \mathrm{h}$. Protein concentration was estimated by measurement of absorbance at 
$280 \mathrm{~nm}$ and the concentration of ethyl $\left[{ }^{3} \mathrm{H}\right.$ ]oleate and $\left[{ }^{14} \mathrm{C}\right]$ oleate in each fraction was measured by standard double-label liquid scintillation counting methods.

\section{RESULTS}

Uncoupling of oxidative phosphorylation in isolated mitochondria induced by fatty acid ethyl esters. Incubation of isolated mitochondria from rabbit ventricle at $25^{\circ} \mathrm{C}$ for $2 \mathrm{~h}$ with ethyl oleate, a fatty acid ethyl ester detected in high concentrations in hearts of humans exposed to alcohol in vivo (21), led to loss of oxidative function in a fashion that was dependent on the concentration of ethyl oleate (Fig. 1). Incubation with $10,25,50,100$, and $200 \mu \mathrm{M}$ ethyl oleate produced progressive decrements in the rate of state III oxygen consumption as well as significant loss of respiratory control. The respiratory control index declined from 7.5 to $6.8,5.9,3.7,3.2$, and 1.0 , respectively. Control mitochondria incubated without ethyl oleate or with vesicles of phosphatidylcholine alone exhibited $<10 \%$ loss of coupling or inhibition of oxygen consumption under the same conditions. Of particular

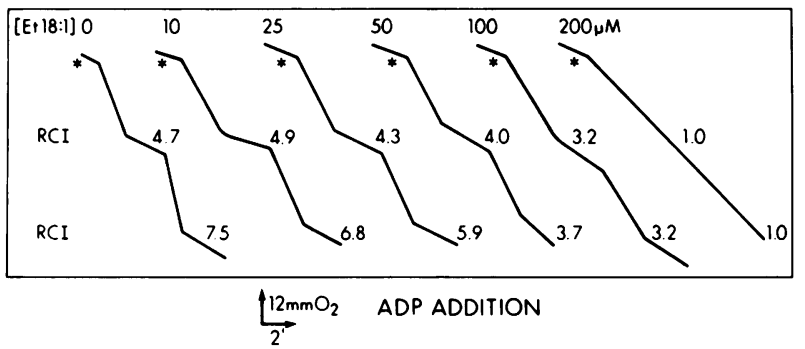

FIGURE 1 Ethyl oleate concentration-dependent inhibition and uncoupling of mitochondrial oxidative phosphorylation. Fresh mitochondria, isolated from rabbit ventricle, were incubated at a concentration of $2 \mathrm{mg} / \mathrm{ml}$ with unilamellar vesicles of egg phosphatidylcholine containing ethyl oleate at the indicated concentrations at $25^{\circ} \mathrm{C}$ in $3.4 \mathrm{ml}$ of buffer containing micromoles of the following components: Tris, 150; phosphate, $37.5 ; \mathrm{Mg}^{++}, 15$; potassium, 300; sucrose, 83 ; EDTA, 20; malate, 67; pyruvate, 67; ADP, 1.25; and BSA, $0.25 \mathrm{mg}$. After $2 \mathrm{~h}$, suspensions were centrifuged at 9,000 $g$ for $10 \mathrm{~min}$ at $4^{\circ} \mathrm{C}$, the supernatant fraction discarded, the mitochondrial pellet resuspended gently, washed once with incubation medium, resuspended in a cuvette, and assayed polarographically for oxygen consumption at $25^{\circ} \mathrm{C}$. State III respiration was initiated by addition of ADP, $1.33 \mathrm{nmol}$, to the suspension (asterisk); the repeated addition (not marked) was performed after state IV respiration achieved steady state. Control recordings with mitochondria incubated with phosphatidylcholine vesicles without ethyl oleate or incubated without any vesicles maintained $>90 \%$ of their original oxidative capacity under these conditions. The respiratory control index $(R C I)$ for each curve shown was calculated as the ratio of the rate of oxygen consumption in state III respiration to that in state IV. Each incubation was performed at least in duplicate. importance, incubation of isolated mitochondria with ethyl oleate, which had been added directly to incubation medium at a concentration of $50 \mu \mathrm{M}$ without prior incorporation into vesicles, resulted in similar uncoupling. However, for convenience and to avoid phase separation with higher concentrations of ethyl oleate, most experiments were performed with ethyl oleate in vesicles of phosphatidylcholine.

When the data are plotted as a function of the concentration of ethyl oleate, it is apparent that the uncoupling process is kinetically complex. Its extent is roughly linear with concentrations of ethyl oleate approaching $\sim 40-50 \mu \mathrm{M}$. However, the extent of uncoupling fails to increase proportionately with higher concentrations (Fig. 2). Even with low concentrations ( 25 and $50 \mu \mathrm{M}$ ethyl oleate), substantial loss of respiratory control occurs. In contrast, no change in mitochondrial respiratory function is evident when incubations are conducted with as much as $200 \mathrm{mM}$ ethanol.

The time course for uncoupling of oxidative phosphorylation that was induced by fatty acid ethyl esters was characterized under conditions in which the concentration of ethyl oleate was held constant at 100 $\mu \mathrm{M}$ (Fig. 3). As the duration of preincubation varied from 15 to $120 \mathrm{~min}$, the uncoupling effect increased linearly. After 120 -min incubation at $25^{\circ} \mathrm{C}$, this concentration of ethyl oleate induced $65-75 \%$ uncoupling. Exposure of mitochondria for $>120 \mathrm{~min}$ led to further uncoupling. Qualitatively similar results were observed with rabbit liver mitochondria, with $\sim 50 \%$ uncoupling after incubation with $100 \mu \mathrm{M}$ ethyl oleate for $60 \mathrm{~min}$.

Binding of ethyl oleate to mitochondria. To determine whether ethyl oleate bound to isolated mitochondria under the incubation conditions employed,

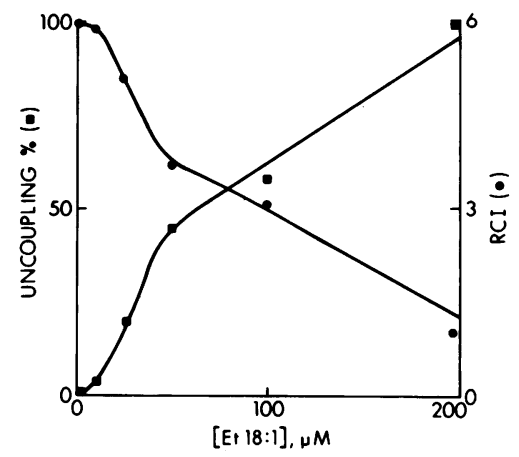

Figure 2 Percentage of mitochondrial uncoupling as a function of the concentration of ethyl oleate. The data from Fig. 1 are plotted graphically, with the percentage of uncoupling calculated assuming that a respiratory control index $(R C I)=1.0$ represents $100 \%$ uncoupling. 


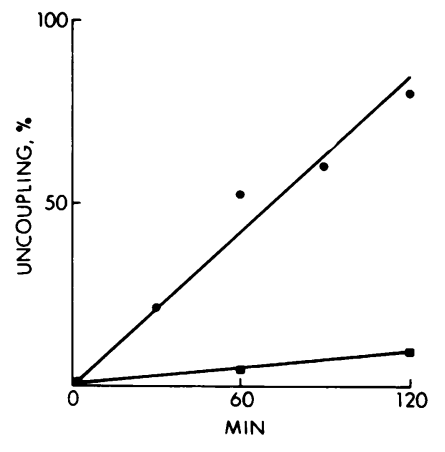

Figure 3 Time course of mitochondrial uncoupling induced by ethyl oleate. The conditions are those described in the legend for Fig. 1 except that the concentration of ethyl oleate was $100 \mu \mathrm{M}$ throughout and that the time of incubation was varied as indicated. Control experiments omitted ethyl oleate. The data were plotted as described in the legend for Fig. 2.

the amount of ethyl $\left[{ }^{3} \mathrm{H}\right]$ oleate bound to mitochondria was quantified. After incubation with ethyl $\left.{ }^{3} \mathrm{H}\right]$ oleate, $100 \mu \mathrm{M}$, for $2 \mathrm{~h}$ at $25^{\circ} \mathrm{C}$, mitochondria were centrifuged at $9,000 \mathrm{~g}$ for $10 \mathrm{~min}$, and then washed three times with incubation medium until no further radioactivity was detected in the wash. After the mitochondria were then recentrifuged, the pellet was extracted for mitochondrial lipids. Binding of ethyl $\left[{ }^{3} \mathrm{H}\right]$ oleate to mitochondria increased linearly with time (Fig. 4), with $\sim 12 \mu \mathrm{M}$ ethyl oleate bound $/ 2 \mathrm{mg}$ mitochondrial protein after exposure for $120 \mathrm{~min}$. Thus, ethyl oleate binds to mitochondria under conditions in vitro that were found to induce mitochondrial dysfunction. Also, results of these experiments demonstrated that $\left[{ }^{3} \mathrm{H}\right]$ oleate derived from ethyl $\left[{ }^{3} \mathrm{H}\right]$ oleate was bound to mitochondria.

To determine the extent of fatty acid ethyl ester binding to mitochondria in intact tissue after myocardial biosynthesis of these lipid metabolites from ethanol, a 2-mm slice of rabbit left ventricle was incubated in $84 \mathrm{mM}\left[{ }^{14} \mathrm{C}\right]$ ethanol, $50 \mathrm{mM}$ phosphate, $\mathrm{pH}$ 7.4, equilibrated with $\mathrm{O}_{2} / \mathrm{CO}_{2}(95: 5)$ at $37^{\circ} \mathrm{C}$ for $2 \mathrm{~h}$; similar experiments with smaller slices of heart provided poor yields of mitochondria. Mitochondria were subsequently isolated from the tissue. Lipids were extracted from the isolated mitochondria and the remainder of the tissue fractions. Of the total of $14 \mathrm{nmol}$ of fatty acid ethyl esters synthesized, the mitochondrial fraction contained $10 \mathrm{nmol}$, or $\sim 72 \%$. Thus, fatty acid ethyl esters synthesized in intact tissue are associated with mitochondria.

Mitochondrial cleavage of ethyl oleate. The time and concentration dependence of uncoupling induced by ethyl oleate and the production of $\left[{ }^{3} \mathrm{H}\right]$ oleate from ethyl $\left[{ }^{3} \mathrm{H}\right]$ oleate by mitochondria suggested that un-

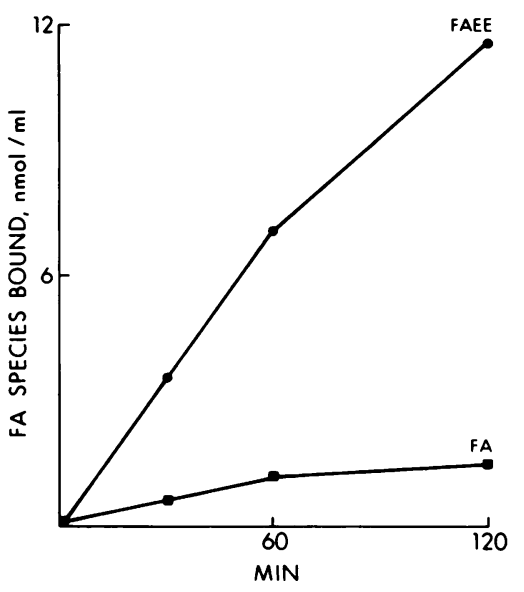

Figure 4 Ethyl $\left[{ }^{3} \mathrm{H}\right]$ oleate binding to isolated mitochondria. Mitochondria, $2 \mathrm{mg} / \mathrm{ml}$, were incubated with ethyl $\left[{ }^{3} \mathrm{H}\right]$ oleate, $100 \mu \mathrm{M}, 500 \mathrm{dpm} / \mathrm{nmol}$, as described in the legend for Fig. 1. At the intervals indicated, the mitochondria were sedimented at $9,000 \times g$ for $10 \mathrm{~min}$ at $4^{\circ} \mathrm{C}$ and washed three times with incubation medium (until only background radioactivity appeared in the last wash). They were then extracted with acetone, the lipids thin-layer chromatographed on silica gel developed with petroleum ether/diethyl ether/ acetic acid (75:5:1) (19), and the amount of labeled ethyl $\left[{ }^{3} \mathrm{H}\right]$ oleate or derived $\left[{ }^{3} \mathrm{H}\right]$ oleate quantified by liquid scintillation spectrometry with yields corrected for recovery of total radioactivity. $F A$, fatty acid; $F A E E$, fatty acid ethyl esters.

coupling might be mediated by cleavage of ethyl oleate to produce free fatty acid, a known uncoupler of mitochondria (29). This interpretation was supported by the observation that when BSA, $440 \mu \mathrm{M}$, was included in the incubation medium, it reduced uncoupling by $100 \mu \mathrm{M}$ ethyl oleate from 75 to $25 \%$ after $2 \mathrm{~h}$ of incubation. Accordingly, the hydrolysis by mitochondria of ethyl oleate was evaluated further.

Incubation of isolated mitochondria, $2 \mathrm{mg} / \mathrm{ml}$, with ethyl $\left[{ }^{3} \mathrm{H}\right]$ oleate, $100 \mu \mathrm{M}$, at $25^{\circ} \mathrm{C}$ resulted in release of $\left.{ }^{3} \mathrm{H}\right]$ oleate into the incubation medium (Fig. 5). The rate of release was linear as a function of time up to 240 min with only $20 \%$ of the initial substrate cleaved in this interval. In the absence of added mitochondria, little if any $\left[{ }^{3} \mathrm{H}\right]$ oleate formed (Fig. 5, lower curve). By varying the initial concentration of ethyl $\left[{ }^{3} \mathrm{H}\right]$ oleate in the reaction mixture, a velocity vs. substrate concentration curve and corresponding Lineweaver-Burk plot were obtained (Fig. 6 and inset). Saturation kinetics were exhibited for $\left[{ }^{3} \mathrm{H}\right]$ oleate release, with a plateau of enzymatic hydrolysis at substrate concentrations $>100 \mu \mathrm{M}$. From the double-reciprocal plot, an apparent Michaelis constant $\left(K_{\mathrm{m}}\right)$ for ethyl oleate of $38 \mu \mathrm{M}$ and catalytic constant of $5 \mathrm{nmol} / \mathrm{h}$ per $\mathrm{mg}$ were obtained. These results demonstrated the pres- 


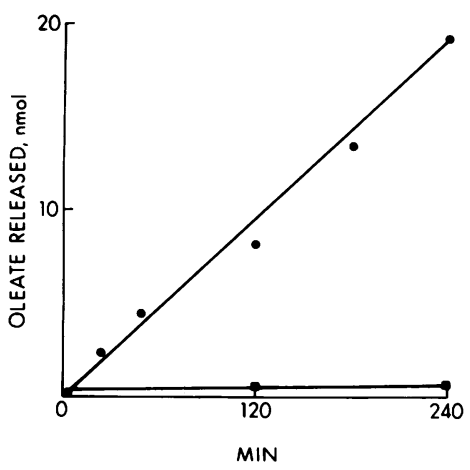

Figure 5 Hydrolysis of ethyl $\left[{ }^{3} \mathrm{H}\right]$ oleate by mitochondria. Mitochondria, $2 \mathrm{mg} / \mathrm{ml}$, were incubated with ethyl $\left[{ }^{3} \mathrm{H}\right]$ oleate, $100 \mu \mathrm{M}$, at $25^{\circ} \mathrm{C}$ (upper curve) under the conditions described in the legend for Fig. 1 and aliquots of the suspension removed at the indicated times. Quantitation of $\left[{ }^{3} \mathrm{H}\right]$ oleate in these aliquots was performed as described in the legend for Fig. 3. Control incubations (lower curve) omitted mitochondria. Data not shown indicated that the rate of $\left[{ }^{3} \mathrm{H}\right]$ oleate released was linear with mitochondrial protein up to $4 \mathrm{mg} / \mathrm{ml}$.

ence of a mitochondrial lipase that can hydrolyze ethyl oleate at concentrations of fatty acid ethyl ester attained in vivo and at rates sufficient to generate micromolar concentrations of free fatty acid.

Fatty acid ethyl ester binding to albumin. Since fatty acid ethyl esters are synthesized in the myocardium from nonesterified fatty acid (20), bind to mitochondria, and may subsequently induce uncoupling of mitochondrial respiration, we evaluated potential interactions of ethyl oleate with BSA to assess the possibility that ethyl esters may function in a pathological shuttle system in which nonesterified fatty acid is mobilized from intracellular binding sites by esterification with ethanol. The subsequent local regeneration by mitochondria of free fatty acid leads to dysfunction. Accordingly, $1 \mathrm{ml}$ of medium containing $29 \mu \mathrm{M}$ defatted serum albumin, $10 \mu \mathrm{M}\left[{ }^{14} \mathrm{C}\right]$ oleate, and $10 \mu \mathrm{M}$ ethyl $\left[{ }^{3} \mathrm{H}\right]$ oleate was incubated at $25^{\circ} \mathrm{C}$ for $10 \mathrm{~min}$, and then chromatographed on Sephadex G-50 equilibrated with incubation medium at $25^{\circ} \mathrm{C}$. In these experiments, the albumin was used as a prototype of intracellular binding proteins. All of the $\left[{ }^{14} \mathrm{C}\right]$ oleate was associated with the protein peak in the resultant effluent. However, only $\sim 34 \%$ of the ethyl $\left[{ }^{3} \mathrm{H}\right]$ oleate (Fig. 7) was associated with the protein peak. It is apparent that a considerable amount of ethyl oleate, but virtually no oleate, remained free. Thus, esterification of free fatty acid to its ethyl ester results in diminished protein binding, which potentially facilitates a net transfer of fatty acid from intracellular protein binding sites to mitochondria.

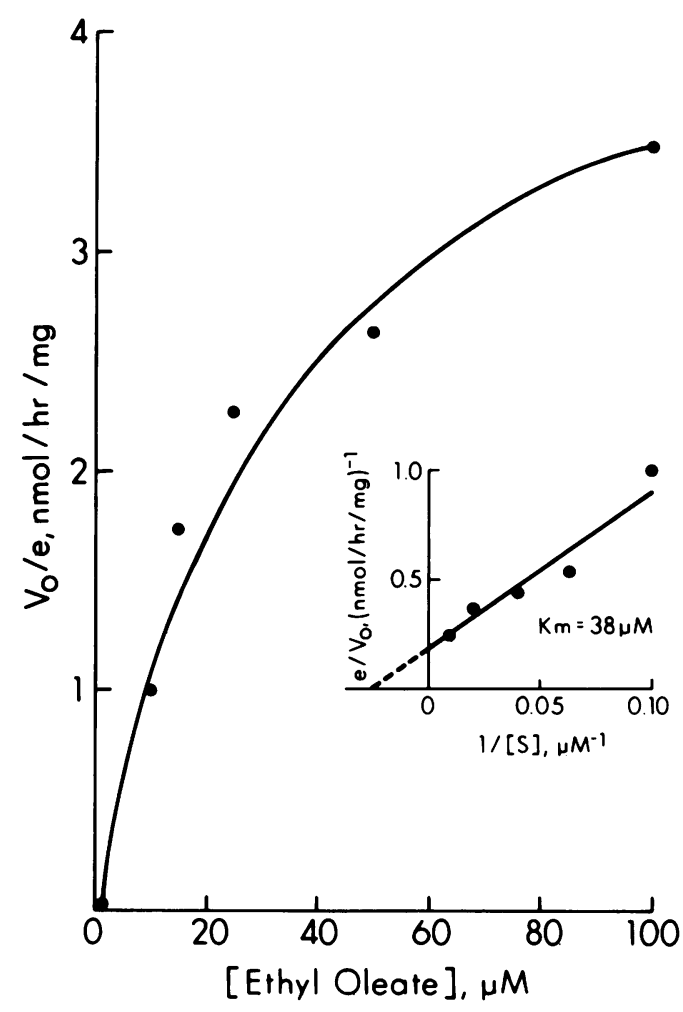

Figure 6 Kinetic plots of cleavage of ethyl $\left[{ }^{3} \mathrm{H}\right]$ oleate by mitochondria. Rates of $\left[{ }^{3} \mathrm{H}\right]$ oleate production from ethyl $\left[{ }^{3} \mathrm{H}\right]$ oleate by isolated mitochondria were obtained from the slope of plots similar to that shown in Fig. 5 obtained at the various ethyl $\left[{ }^{3} \mathrm{H}\right]$ oleate concentrations. Results were expressed as nanomoles $\left[{ }^{3} \mathrm{H}\right]$ oleate formed per hour per milligram mitochondrial protein. A double-reciprocal plot is shown in the inset, with an apparent $K_{m}$ value calculated from the $x$-axis intercept.

\section{DISCUSSION}

The data presented demonstrate that fatty acid ethyl esters, myocardial metabolites of ethanol, induced mitochondrial dysfunction characterized by impaired respiratory coupling of oxidative phosphorylation and, at higher concentrations, diminished rates of state III oxygen consumption. Incubation of mitochondria isolated from rabbit ventricle with ethyl oleate resulted in a time- and ethyl oleate concentration-dependent loss of mitochondrial function (Figs. 1-3) at concentrations of ethyl esters that are known to occur in human myocardium (21). Uncoupling correlated with binding of ethyl oleate to mitochondria (Fig. 4) and with hydrolysis of the ester by mitochondria to form oleate (Figs. 5 and 6), a known uncoupler of oxidative phosphorylation (29). Because fatty acid ethyl esters are formed in myocardium principally from nonesterified fatty acid (20), since ethyl esters are bound less 


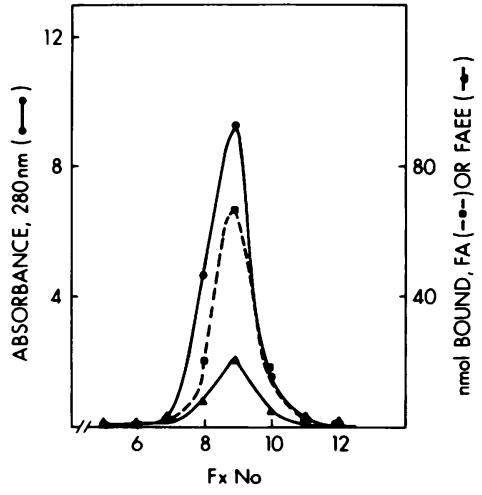

Figure 7 Relative binding affinities of $\left[{ }^{14} \mathrm{C}\right]$ oleate and ethyl $\left[{ }^{3} \mathrm{H}\right]$ oleate to BSA. $10 \mathrm{nmol}$ of $\left[{ }^{14} \mathrm{C}\right]$ oleate and $10 \mathrm{nmol}$ ethyl $\left[{ }^{3} \mathrm{H}\right]$ oleate were incubated with $29 \mathrm{nmol}$ defatty BSA in $1 \mathrm{ml}$ at $25^{\circ} \mathrm{C}$ for $10 \mathrm{~min}$ in the same medium as that described in the legend for Fig. 1. The reaction mixture was then gel filtered through Sephadex G-50 $(1.6 \times 30 \mathrm{~cm})$, equilibrated with the same buffer. Fractions of $1 \mathrm{ml}$ were collected at 25 $\mathrm{ml} / \mathrm{h}$. Protein, $\left[{ }^{14} \mathrm{C}\right]$ oleate, and ethyl $\left[{ }^{3} \mathrm{H}\right]$ oleate concentrations were determined as described in Methods. Approximately $100 \%$ of the $\left[{ }^{14} \mathrm{C}\right]$ oleate co-eluted with the protein, but only $34 \%$ of the ethyl $\left[{ }^{3} \mathrm{H}\right]$ oleate co-eluted. The remainder of the ethyl ester eluted in a broad peak in the included volume. $F A$, fatty acid; $F A E E$, fatty acid ethyl ester.

readily to protein than are nonesterified fatty acids (Fig. 7), and because fatty acid ethyl esters synthesized in myocardial tissue slices bind substantially to mitochondria, fatty acid ethyl esters may act in a toxic fatty acid shuttle to induce mitochondrial dysfunction in vivo after prolonged alcohol abuse. A mechanism for such a shuttle is depicted in Fig. 8.

Under physiological conditions, the amount of nonesterified fatty acid (total intracellular concentration in the range of $250-500 \mathrm{nmol} / \mathrm{g}[20,30])$ that is $\mathrm{ki}$ netically free is probably quite small. Intracellular binding sites for nonesterified fatty acid have been identified, such as fatty acid binding protein (31). As shown in Fig. 8, they may protect mitochondria and other organelles from damage by maintaining the concentration of free fatty acid at low levels. However, as shown in the lower panel of Fig. 8, after consumption of ethanol, nonesterified fatty acid becomes converted to fatty acid ethyl ester by a cytoplasmic esterase (20). The esterified compounds can accumulate in concentrations as high as $115 \mu \mathrm{M}$ in human myocardium (21). Since fatty acid ethyl esters are neutral molecules, they bind less readily to proteins, such as albumin (Fig. 7), and also possibly to fatty acid binding proteins, which bind molecules that are negatively charged at physiological $\mathrm{pH}$. The fatty acid moiety can then traverse the intracellular space due to its covalent bond to ethanol and the altered binding specificity of ethyl esters. Mitochondria and other hydro-

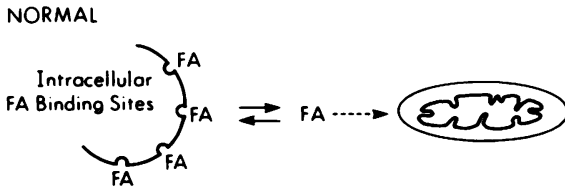

$\mathrm{ErOH}$

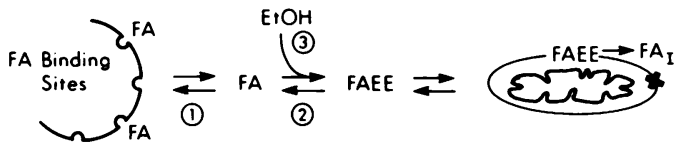

FigURE 8 Schematic model of fatty acid ethyl ester (FAEE) acting as a toxic fatty acid $(F A)$ shuttle inducing mitochondrial dysfunction. Above: Intracellular, nonesterified fatty acid is depicted bound to intracellular fatty acid binding sites and hence kinetically unavailable (dashed arrow) for potentially deleterious interactions with mitochondrial membranes. Below: Ethanol ingestion leading to formation of FAEE may result in net transfer of fatty acid from the intracellular fatty acid binding sites to the neutrally charged esterified form that is bound only poorly to protein, but bound significantly to mitochondria. Such net transfer could give rise to production of nonesterified fatty acid intrinsic to mitochondria $\left(F_{1}\right)$ that could then induce mitochondrial dysfunction $(X)$, an effect to which FAEE could also directly contribute. The circled numbers refer to sites of potential modulation of the processes schematized and discussed in the text.

phobic moieties can readily accumulate the transported and transformed fatty acid, judging from our findings that $72 \%$ of synthesized fatty acid ethyl ester were bound to mitochondria. Although experiments with even 2-mm thick tissue slices may not reflect biological events in whole organs in vivo in a one-to-one fashion, it is clear that some binding of ethyl esters to mitochondria does occur. Once they become attached to the mitochondrial surface, the ethyl esters may exert deleterious effects on mitochondrial function through several mechanisms (Fig. 8).

Mitochondria contain an enzyme that can cleave ethyl oleate to form fatty acid and ethanol (Fig. 8). Fatty acid itself is a known inhibitor and uncoupler of mitochondrial oxidation in concentrations as low as $5 \mu \mathrm{M}$ (29). On the other hand, since fatty acid ethyl esters bind to mitochondria, they may, of course, directly affect mitochondrial function, especially over long intervals. Such direct modulation of function would not be unreasonable since incorporation of a neutral lipid into the mitochondrial membrane could alter its permeability to other ions that are important in oxidative phosphorylation.

Several factors may modulate fatty acid ethyl esterinduced mitochondrial damage (Fig. 8, encircled numbers) in subjects exposed to ethanol. First, factors that regulate the equilibrium concentration of kinetically free fatty acid could be important, because enzymatic 
conversion to ethyl esters might be affected by them. Second, the activity of intracellular enzyme(s) that synthesize fatty acid ethyl esters may be influenced both by small molecule effectors or potentially by isoenzymes with inherent differences in catalytic properties. Third, the amount of ethanol ingested and its rate of clearance would modify the concentration of ethanol in myocardium available for esterification. Fourth, the rates at which nonesterified fatty acid is regenerated in or near the mitochondria membrane may be variable. Modification of these and other metabolic factors is probable. Thus, the overall effect on mitochondria may be different from individual to individual because of genetic or environmental factors or both. Furthermore, since alcohol abuse is chronic, metabolic insults to myocardial mitochondria may occur over long intervals. Thus, the reparative reserve of mitochondria may be critical.

For many years it has been assumed that myocardium does not metabolize ethanol $(6,9)$. Thus, unlike the liver, for which at least three metabolic pathways for ethanol oxidation have been identified that are quantitatively more significant than hepatic fatty acid ethyl ester formation, it has been difficult to relate ethanol ingestion to cardiac disease because of the lack of an intermediary metabolic link. Results of our studies have demonstrated that fatty acid ethyl esters are formed in isolated, perfused hearts (19). The existence of such compounds in biological systems has been shown previously, but a number of important points still merit consideration. The first description of fatty acid ethyl esters was made in 1963 (32) in rats injected with $\left[{ }^{14} \mathrm{C}\right]$ ethanol. At that time the extant methods used included lipid extraction consisting of Waring blender (Waring Products Div., Dynamics Corp. of America, New Hartford, CT) homogenization of the entire animal in 50\% ethanol and a 2-d processing step at room temperature. Since chemical esterification is known to occur under these circumstances (33), the actual extent of in vivo formation of fatty acid ethyl esters is difficult to estimate. Of interest, in another study, fatty acid ethyl esters were identified in Rhizopus arrhizus (34), but no enzymatic pathway for their formation was identified. In vitro production of fatty acid ethyl esters was noted initially in 1973 in liver microsomes (35) and subsequently confirmed (36). In view of the widespread use of methanol as a competing nucleophile for water in the study of numerous proteases (37) to form methyl esters, the quantitative importance of the finding that ethanol in vitro may give rise to ethyl esters is difficult to assess, especially when $40 \mu \mathrm{M}$ palmitoyl-Coenzyme A, a supraphysiological concentration, was used in the assays. Subsequent studies in our laboratory demonstrated that fatty acid ethyl esters are indeed formed in vivo $(21,38)$ and that fatty acid ethyl ester synthesis uses primarily nonesterified fatty acid as a substrate (20) at their respective physiological concentrations. Purified cholesterol esterase from pancreas was demonstrated to rapidly catalyze the formation of fatty acid ethyl esters from nonesterified fatty acid and ethanol (20). Therefore, it was hypothesized that this or a very similar enzyme catalyzed their formation in the heart. Subsequently, we purified over a 1,000-fold to near homogeneity from rabbit myocardium an enzyme that rapidly synthesizes fatty acid ethyl esters from ethanol and nonesterified fatty acid (39). Significantly, heart is much more active than liver in overall fatty acid ethyl ester formation and accumulation (38), while skeletal muscle is essentially inactive. Studies have also shown that fatty acid ethyl esters accumulate in human myocardium with $t_{1 / 2}$ substantially exceeding that of ethanol itself $(21,38)$. The present results indicated that fatty acid ethyl esters can induce mitochondrial dysfunction.

Mitochondrial dysfunction has been implicated strongly in the syndrome of alcohol-induced heart disease. In 1917, it was shown that oxygen consumption is increased after even moderate ingestion of ethanol in some individuals (10). Over the past $20 \mathrm{yr}$, light and electron microscopic changes in mitochondrial structure and function have been documented in myocardium from animals $(11,12)$ and human subjects $(13$, 14) exposed to ethanol. After a lag period of ethanol exposure in vivo, mitochondria isolated from the heart exhibit impaired rates of oxygen consumption, diminished respiratory control, and reduced $\mathrm{P} / \mathrm{O}$ ratios (1518). Although such changes may regress in vivo with cessation of exposures to ethanol, reversibility is not immediate but requires up to 8 wk (17).

The results of this study suggest that fatty acid ethyl esters, myocardial metabolites of ethanol, may contribute to the mitochondrial dysfunction associated and possibly pathogenetically involved in alcohol-induced heart disease through several potential mechanisms including induction of a toxic fatty acid shuttle in the heart.

\section{ACKNOWLEDGMENTS}

Appreciation is expressed to Michael Hartman for his technical assistance and to Dr. J. Saffitz and E. Williams for their assistance with the electron microscope.

The research for this paper was supported in part by $\mathrm{Na}$ tional Institutes of Health grant 1-R01-HL30152.

\section{REFERENCES}

1. Bridgen, W., and J. Robinson. 1964. Alcoholic heart disease. Br. Med. J. 2:1283-1289.

2. Schwartz, L., K. A. Semple, and E. D. Wigle. 1975. Severe alcoholic cardiomyopathy reversed with abstention from alcohol. Am. J. Cardiol. 36:963-966. 
3. Demakis, J. G., A. Prostzey, S. H. Rahimtoola, M. Jamil, G. C. Sutton, K. M. Rosen, R. M. Gunnar, and J. R. Tobin. 1974. The natural course of alcoholic cardiomyopathy. Ann. Intern. Med. 80:293-297.

4. Regan, T. J., G. Koroxenidis, C. B. Moschos, H. A. Oldewurtel, P. H. Lehan, and H. K. Hellems. 1966. The acute metabolic and hemodynamic responses of the left ventricle to ethanol. J. Clin. Invest. 45:270-280.

5. Regan, T. J., P. O. Ettinger, B. Haider, S. S. Ahmed, H. A. Oldewurtel, and M. M. Lyons. 1977. The role of ethanol in cardiac disease. Annu. Rev. Med. 28:393-409.

6. Lochner, A., R. Cowley, and A. J. Brink. 1969. Effect of ethanol on metabolism and fuction of perfused rat heart. Am. Heart J. 78:770-780.

7. Kikuchi, T., and K. J. Kako. 1970. Metabolic effects of ethanol on the rabbit heart. Circ. Res. 26:625-634.

8. Segal, L. D., S. V. Rindig, and D. T. Mason. 1979. Left ventricular dysfunction of isolated working rat hearts after chronic alcohol consumption. Cardiovasc. Res. 13:136-146.

9. Gailis, L., and M. Verdy. 1971. The effect of ethanol and acetaldehyde on the metabolism and vascular resistance of the perfused heart. Can. J. Biochem. 49:227-233.

10. Higgins, H. 1917. Effect of alcohol on the respiration and the gaseous metabolism in man. J. Pharmacol. Exp. Ther. 9:441-472.

11. Segel, L. D., S. V. Rendig, V. Choquet, K. Chacho, E. A. Amsterdam, and D. T. Mason. 1975. Effects of chronic graded ethanol consumption on the metabolism, ultrastructure and mechanical function of the rat heart. Cardiovasc. Res. 9:649-663.

12. Brigden, W. 1972. Alcoholic cardiomyopathy. In Cardiomyopathy. G. E. Burch, editor. F. A. Davis Co., Philadelphia. 131-147.

13. Ferrans, V. J., R. G. Hibbs, D. G. Weilbaecher, W. C. Black, J. J. Walsh, and G. E. Burch. 1965. Alcoholic cardiomyopathy: a histochemical study. Am. Heart J. 69:748-765

14. Alexander, C. S. 1967. Electron microscopic observations in alcoholic heart disease. Br. Heart J. 29:200-206.

15. Cederbaum, A. I., C. S. Leiber, and E. Rubin. 1974. Effect of chronic alcohol treatment on mitochondrial functions. Arch. Biochem. Biophys. 165:560-569.

16. Pachinger, O. M., H. Tillmanns, J. C. Mao, J.-M. Fauvel, and R. J. Bing. 1973. The effect of prolonged administration of ethanol on cardiac metabolism and performance in the dog. J. Clin. Invest. 52:2690-2696.

17. Weishaan, R., J. S. M. Sarma, Y. Maruyama, R. Fischer, S. Bertuglia, and R. J. Bing. 1977. Reversibility of mitochondrial and contractile changes in the myocardium after cessation of prolonged ethanol intake. Am. J. Cardiol. 40:556-562.

18. Bottenus, R. E., P. I. Spach, S. Filns, and C. C. Cunningham. 1982. Effect of chronic ethanol consumption on energy-linked processes associated with oxidative phosphorylation. Biochem. Biophys. Res. Commun. 105:1368-1373.

19. Lange, L. G., S. R. Bergmann, and B. E. Sobel. 1981. Identification of fatty acid ethyl esters as products of rabbit myocardial ethanol metabolism. J. Biol. Chem. 256:12968-12973.

20. Lange, L. G. 1982. Nonoxidative ethanol metabolism: formation of fatty acid ethyl esters by cholesterol esterase. Proc. Natl. Acad. Sci. USA 79:3954-3957.

21. Lange, L. G., and B. E. Sobel. 1982. Accumulation of unique metabolites, fatty acid ethyl esters, in human myocardium after consumption of ethanol. Circulation 66(Suppl. II):214. (Abstr.)

22. Cleland, K. W., and E. C. Slater. 1953. Respiratory granules of heart muscle. Biochem. J. 53:547-559.

23. Chance, B., and B. Hagoihara. 1960. Initiation of succinate oxidation in aged pigeon heart mitochondria. Biochem. Biophys. Res. Commun. 3:1-9.

24. Sobel, B. E., A. Sjoerdsma, E. Jequier, and W. Lovenberg. 1966. Effect of catecholamines and adrenergic blocking agents on oxidative phosphorylation in rat heart mitochondria. Circ. Res. 19:1050-1058.

25. Chance, B., and G. R. Williams. 1955. Respiratory enzymes in oxidative phosphorylation. J. Biol. Chem. 217:382-387.

26. Wakabayashi, T., E. F. Korman, and D. E. Green. 1971. On the structure of biological membranes: the doubletiered pattern. J. Bioenerg. 2:233-247.

27. Lowry, O. H., N. J. Rosebrough, A. L. Farr, and R. J. Randall. 1951. Protein measurement with the folin phenol reagent. J. Biol. Chem. 193:265-275.

28. DeKruijff, B., P. R. Cullis, and G. K. Radda. 1976. Outside-inside distributions and sized of mixed phosphatidylcholine-cholesterol vesicles. Biochim. Biophys. Acta. 436:729-740.

29. Borst, P., J. A. Loos, E. J. Christ, and E. C. Slater. 1962. Uncoupling activity of long chain fatty acids. Biochim. Biophys. Acta. 62:509-517.

30. Kramer, J. K. G., and H. M. Hulan. 1978. A comparison of procedures to determine free fatty acids in rat heart. J. Lipid Res. 19:103-106.

31. Ockner, R. K., and J. A. Manning. 1974. Fatty acid binding protein in small intestine. J. Clin. Invest. 54:326338 .

32. Goodman, D. S., and D. Deykin. 1963. Fatty acid ethyl ester formation during ethanol metabolism in vivo. Proc. Soc. Exp. Biol. Med. 113:65-67.

33. Lough, A. K., L. Felinsky, and G. A. Garton. 1962. The production of methyl esters of fatty acids as artifacts during the extraction or storage of tissue lipids in the presence of methanol. J. Lipid Res. 3:478-481.

34. Laseter, J. L., and J. D. Weete. 1971. Fatty acid ethyl esters of Rhizopus arrhizus. Science (Wash. DC). 172:864865.

35. Grigor, M. R., and I. C. Bell. 1973. Synthesis of fatty acid esters of short chain alcohols by an acyltransferase in rat liver microsomes. Biochim. Biophys. Acta. 306:2630.

36. Polokoff, M. A., and R. M. Bell. 1978. Limited palmitoylCoA penetration into microsomal vesicles as evidenced by a highly latent ethanol acyltransferase activity. $J$. Biol. Chem. 253:7173-7178.

37. Dixon, M., and E. C. Webb. 1979. Enzymes. Academic Press, Inc., New York. 3rd edition. 303.

38. Lange, L. G., and B. E. Sobel. 1983. Myocardial metabolites of ethanol. Circ. Res. 52:479-482.

39. Mogelson, S., and L. G. Lange. 1983. Purification of fatty acid ethyl esters synthetase from rabbit myocardium. FASEB (Fed. Am. Soc. Exp. Biol.) Monogr. (Abstr.) 42:1687. 